\title{
sciendo
}

10.2478/AMB-2020-0029

\section{GAUCHER DISEASE TYPE I: A CASE REPORT}

\author{
D. Nikolova', ${ }^{1,2}$ A. Yordanov ${ }^{3}$, V. Damyanova ${ }^{1,2}$, A. Yavorova ${ }^{3}$, A. Radinov ${ }^{3}$ \\ 'Department of Medical Genetics, Medical University - Sofia, Bulgaria \\ ${ }^{2}$ Genetic Laboratory, UMHAT "Sv. Ivan Rilski" - Sofia, Bulgaria \\ ${ }^{3}$ Clinic of Hematology, UMHAT "Sv. Ivan Rilski“ - Sofia, Bulgaria
}

\begin{abstract}
Gaucher disease $(G D)$ is a multi-systemic disease with a low population frequency. It is a lysosomal storage disorder (LSD) that causes accumulation of glucocerebroside in the so called Gaucher cells predominantly in areas like the spleen, liver and bone marrow. Type I GD (GDI) is the most common form and usually does not involve the brain and the spinal cord. The symptoms can range from mild to severe and may appear anytime from childhood to adulthood. Diagnostics can often be challenging and imposes looking at person's medical history, symptoms, physical exam, and laboratory test results. We present a difficult to diagnose case of a 34-year-old woman admitted to the Clinic of Hematology, "Sv. I. Rilski" hospital with splenomegaly, normal laboratory findings and non-enlarged liver. She didn't show focal neurological symptoms. A series of tests were assigned including genetic targeted analysis. The case is an example of a rare genetic disease with mild clinical symptoms. Diagnosis of Gaucher disease, type I was confirmed by measurement of a GBA enzyme activity and identification of mutations in the GBA gene inherited in an autosomal recessive manner. Thanks to the efforts of the clinical team, the assignment of adequate clinical and laboratory tests and their correct interpretation, the patient was subjected to enzyme replacement therapy (ERT). Although the diagnosis was settled relatively late (at 34 years of age), the correct therapy slowed down the invalidation and improved the quality of life of the patient.
\end{abstract}

Key words: Gaucher disease, genetic test, diagnostics

Corresponding author: Assist. Prof. Dragomira Nikolova, PhD, Clinic of Hematology, UMHAT „Sv. Ivan Rilski", 1612 Sofia, Bulgaria, e-mail: dmb@abv.bg

\section{INTRODUCTION}

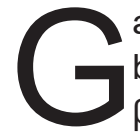
aucher disease is a rare multi-systemic metabolic disease that results from a deficiency of $\beta$-glucosidase activity accompanied by large deposits of glucoserebroside inside the cells. In the general population, the incidence of this syndrome is extremely low (between 1 in 50,000 and 1 in 100,000), but in some populations it is significantly more common (e.g. Ashkenazi Jews), where it ranges from 1 in 500 to 1 in 1000 patients. The gold standard for establishment of the diagnosis of Gaucher disease is the presence of less than $15 \%$ of the average normal activity of the enzyme $\beta$-glucosidase in leukocytes, which is usually accompanied by increased activity of chitotriosidase (chitinase I) [1]. Gaucher is a typical lysosomal "disease of accumulation" most commonly associated with hepatosplenomegaly and cytopenia [2]. Progressive cytopenia is interpreted as an indication of an advanced disease and imposes a necessity for the initiation of specific therapy. Bone marrow evaluation is not routinely performed in Gaucher disease, and for that reason other causes of cytopenia such as myelodysplastic syndrome (MDS) may be missed [2]. Other forms of Gaucher disease are running simultaneously with MDS, but they are quite rare. 
Gaucher disease has three forms, the first of which is the most common one. Most individuals are diagnosed accidentally; some of them remain asymptomatic and usually have no neuronal manifestations. The other two (Gaucher type 2 and 3) are neurodegenerative, acute and subacute forms, respectively. After the biochemical measurement of metabolic activity, the confirmation of the clinical diagnosis is made by a genetic test performed by a reference laboratory specialized in hereditary metabolic diseases.

The aim of this report was to present a case of a woman with mild clinical symptoms whose diagnosis was set at the age of 34 years; albeit the diagnosis was established relatively late, the treatment of the patient with a targeted therapy drug gave positive results.

\section{CASE REPORT}

A 34-year-old woman was admitted to the Clinic of Hematology at the University Hospital "Sv. Ivan Rilski" for diagnostic specification on the basis of previously established splenomegaly $(172 / 65 \mathrm{~mm})$. She did not report other concomitant illnesses and/or allergies, but did mention mild fatigue and left-sided discomfort. Upon clinical examination, palpation detected an enlarged spleen reaching the upper umbilical horizontal, while the liver was not palpated enlarged. No focal neurological symptoms were detected and the patient was afebrile at admission.

The patient was assigned a series of additional tests including clinical laboratory tests, abdominal ultrasound, magnetic resonance imaging, bone marrow biopsy, biochemical and genetic analysis. The patient signed a written informed consent and agreed the case to be reported in compliance with all confidentiality rules. Clinical and laboratory studies didn't show significant deviations from reference values. The only parameter outside of the ranges was uric acid which was slightly elevated (Table 1).

Abdominal ultrasound showed normal size liver with homogeneous structure; gallbladder without concretions, normal portal vein, non-dilated biliary tract, pancreas of normal size and structure. Abdominal ultrasound confirmed the clinical examination, showing an enlarged spleen (170/62 mm) and hyperechogenic structure. The MRI examination of the lumbar segment of the spine did not show any pathological changes related to bone marrow edema. Severe focal marrow edema (third degree) was detected in both femurs, affecting the entire diaphyses, as well as the metaphyses of both bones. No deformities were detected in the distal divisions of the two femurs.
Table 1. Clinical and laboratory parameters of the patient

\begin{tabular}{|c|c|c|c|}
\hline Parameter & Value & Units & Reference ranges \\
\hline WBC & 4,83 & $10^{\wedge} 9 / \mathrm{L}$ & $3.50-10.50$ \\
\hline RBC & 4,63 & $10^{\wedge} 12 / \mathrm{L}$ & $3.70-5.30$ \\
\hline HGB & 136 & $\mathrm{~g} / \mathrm{L}$ & 120160 \\
\hline HTC & 0,422 & $\mathrm{~L} / \mathrm{L}$ & $0.360-0.480$ \\
\hline MCV & 91,2 & $f L$ & $80.0-96.0$ \\
\hline $\mathrm{MCH}$ & 29,3 & pg & $27.0-33.0$ \\
\hline $\mathrm{MCHC}$ & 322 & $g / L$ & $300-360$ \\
\hline PLT & 155 & $10^{\wedge} 9 / \mathrm{L}$ & $130-360$ \\
\hline LYM & 26,8 & $\%$ & $20.0-48.0$ \\
\hline MONO & 6,1 & $\%$ & $1.0-11.0$ \\
\hline EO & 3 & $\%$ & Up to 6.5 \\
\hline BASO & 0,7 & $\%$ & Up to 2.0 \\
\hline NEUT & 61,5 & $\%$ & $40.0-70.0$ \\
\hline LYM & 1,29 & $x 10^{\wedge} 9 / \mathrm{L}$ & $1.00-4.00$ \\
\hline MONO & 0,29 & $x 10^{\wedge} 9 / \mathrm{L}$ & Up to 0.80 \\
\hline EO & 0,15 & x10^9/L & Up to 0.50 \\
\hline BASO & 0,04 & $x 10^{\wedge} 9 / \mathrm{L}$ & Up to 0.14 \\
\hline NEUT & 2,97 & $x 10^{\wedge} 9 / L$ & $2.00-7.00$ \\
\hline RDW & 14,6 & & \\
\hline MPV & 9,5 & $f \mathrm{~L}$ & $6.3-12.5$ \\
\hline creatinine & 70 & $\mu \mathrm{mol} / /$ & Up to 96.00 \\
\hline Uric acid & 402.60 & $\mu \mathrm{mol} / /$ & $142.00-340.00$ \\
\hline ALAT & 16,88 & $\mathrm{U} / \mathrm{I}$ & Up to 33.00 \\
\hline $\mathrm{LDH}$ & 256 & $U / L$ & Up to 460 \\
\hline
\end{tabular}

Abbr.: WBC - white blood cells, RBC - red blood cells, HGB - hemoglobin, HTC (hematocrit, MCV (mean corpuscular volume, $\mathrm{MCH}$ (mean corpuscular hemoglobin), MCHC (mean corpuscular hemoglobin concentration), PLT (platelets), LYM (lymphocytes), MONO (monocytes), EO (eosinophil), BASO (basophils), NEUT (neutrophils), RDW (red cell distribution width), MPV (mean platelet volume), ALAT (alanine aminotransferase), LDH (lactic acid dehydrogenase)

The bone marrow biopsy showed optimum material of spongy bone with hypocellular bone marrow consistent with the age of the patient, at the expense of a cohesive, macrophage, mainly epitheloid composition with extensive amphophilic cytoplasms and a small laterally located nucleus. The patient was PAS (+) and Gomori staining normal proved by $\mathrm{IHC}$ analysis.

Tandem mass spectrometry was performed with a dry drop of blood. The activity of the enzymes $\beta$-glucocerebrosidase and acid sphingomyelinase was measured. The results are presented in Table 2. Acid sphingomyelinase showed very low activity 
(below the reference limit). Since Gaucher disease could not be ruled out in this case, a genetic analysis was assigned.

Table 2. Biochemical activity of the patient's enzymes $\beta$-glucocerebrosidase and acid sphingomyelinase

\begin{tabular}{|l|c|c|c|}
\hline & Result & \multicolumn{1}{|c|}{ Units } & $\begin{array}{c}\text { Reference } \\
\text { range }\end{array}$ \\
\hline$\beta$-glucocerebrosidase & 0.4 & $\mu \mathrm{mol} / / / \mathrm{h}$ & $>2.5$ \\
\hline Acid sphingomyelinase & 8.1 & $\mu \mathrm{mol} / / \mathrm{h}$ & $>0.9$ \\
\hline
\end{tabular}

The diagnosis was confirmed by targeted genetic analysis. A homozygous mutation c. [1226A > G] ( $p$. [Asn409Ser]; [Asn409Ser]) was found in the GBA gene ( $\beta$-glucocerebrosidase enzyme coding gene).

\section{DISCUSSION}

The diagnosis of Gaucher disease is based on clinical symptoms and laboratory testing. The diagnosis is suspected in individuals with bone problems, enlarged liver and spleen (hepatosplenomegaly), changes in red blood cell levels, bleeding and hematomas due to low platelet counts, sometimes (depending on the form of the disease) changes in the neuronal functions (https://www.genome.gov/Genetic-Disorders/ Gaucher-Disease). In our case, the patient had only splenomegaly and normal clinical laboratory findings. Blood cells were normal, there was no liver enlargement; no pathological changes in the liver enzymes were found (see Table 1). The patient, though, was positive for PAS staining. The method has been used to detect polysaccharides such as glycogen and mucous substances such as glycoproteins, glycolipids and mucins in tissues. Macrophages are also stained positive by the PAS method because of the accumulation of glucose cerebroside. It does not stain sphingomyelin and other compounds containing glycol groups (eg in Neumann-Peak disease) [3]. Neumann-Peak's disease was also ruled out in subsequent biochemical analysis showing normal activity of the enzyme acid sphingomyelinase (Table 2).

The diagnosis of Gaucher disease requires mainly biochemical analysis of the enzyme activity as well as DNA analysis for the presence of mutational alleles in the GBA gene in homozygous state. Bone marrow biopsy is not obligatory to settle the diagnosis. In cases where a specific gene mutation has been previously identified in the family, genetic testing can be performed to identify the carriers in the pedigree. However, it is often impossible to predict the clinical status and development of the disease based on DNA testing alone due to the variable expressivity of the mutation.

The mutation found is a common pathogenic missense mutation and proves the presence of Gaucher disease. To date, 36 mutations in the GBA gene causing the disease have been described so far [4]. These are point mutations, deletions, gene fusions, and recombinations in a homologous pseudogene that has been described in the gene encoding glucose cerebrosidase [5]. They can be classified as lethal, severe or mild depending on the related phenotypic effect. Mild mutations are not associated with neuronal symptoms and cause type I Gaucher disease, even if they are inherited in a combination with a lethal or severe mutation. Such a mutation is the one found in the patient 1226G (Asn409Ser/Asn409Ser). Together with four other mutations (84GG, inv $2(+1)$, $1448 \mathrm{C}, 1297 \mathrm{~T})$, it represents about $75 \%$ of the identified pathological alleles [6] in patients of non-Jewish origin. Recently, the attention has also been focused on the relationship between the carriership of mutations in GBA and the development of Parkinson's disease (PD) [7]. This does not mean that any carrier will develop PD. It is estimated that only $9.1 \%$ of the carriers of mutations in the GBA gene develop PD. According to some studies, the penetrance of $P D$ in patients with Gaucher disease is $30 \%$ at 80 years of age [8]. Carrying a mutation in one allele of the GBA gene increases the risk of PD 5-fold, and in both alleles (homozygotes) - 10-20 times [9, 10].

Enzyme replacement therapy (ERT) was approved for Gaucher disease in 1991 and involves intravenous infusion of a modified enzyme which compensated the enzyme deficiency, minimizes symptoms and long-term damage to the patient, and balances low levels of glucocerebrosidase (https://www.gaucherdisease.org). Infusions typically last between 1-2 hours. ERT includes three FDA-approved drugs: Cerezyme (imiglucerase), VPRIV (velaglucerase alfa) and Elelyso (taliglucerase alfa).

Upon diagnosis, the patient was switched to cerezyme infusion, which is indicated for long-term therapy in children and elderly patients with Gaucher Type I disease. The patient responded well without significant side effects.

\section{CONCLUSION}

Genetic diseases often do not exhibit typical clinical symptoms due to the variable expressivity of the mutation and its type. We are presenting a case of a woman with Gaucher disease who exhibited mild 
form of type I disease, which made the case difficult to diagnose. The biochemical analysis, as well as the subsequent genetic one, both confirmed the clinical diagnosis. The homozygous carrier of the p.1226G mutation defined the disease as Gaucher, type I. Although later in life (in mid-thirties), the case was properly diagnosed and the patient received disease-suitable therapy that reduced symptoms and improved her quality of life.

Acknowledgements* $D$. Nikolova and A. Yordanov contributed equally to the preparation of the manuscript.

Disclosure summary: The authors have nothing to disclose.

\section{REFERENCES:}

1. Fateen E, Abdallah ZY. Twenty-five years of biochemical diagnosis of Gaucher disease: the Egyptian experience. Heliyon, 2019, 5(10):e02574.

2. Ruchlemer R MM, Zimran A. Gaucher disease, myelodysplastic syndrome and ICUS. Blood Cells Mol Dis., 2020, 80(102373).
3. Pernick, N. Bone marrow - nonneoplastic systemic disorders. Gaucher disease. Pathology Outlines, 2019.

4. Beutler E, Demina A, Gelbart T. Glucocerebrosidase mutations in Gaucher disease. Mol Med, 1994, 1(1):82-92.

5. Beutler E. Gaucher disease as a paradigm of current issues regarding single gene mutations of humans. Proc Natl Acad Sci U S A, 1993, 90(12):5384-90.

6. Beutler E, Gelbart T. Gaucher disease mutations in non-Jewish patients. Br J Haematol, 1993., 85(2):401-5.

7. Riboldi G, Di Fonzo AB. Gaucher Disease, and Parkinson's Disease: From Genetic to Clinic to New Therapeutic Approaches. Cells, 2019, 8(4), 364. doi: 10.3390/cells8040364.

8. Anheim M, Elbaz A, Lesage $S$ et al. Penetrance of Parkinson disease in glucocerebrosidase gene mutation carriers. Neurology, 2012, 78(6):417-20.

9. McNeill A, Duran R, Proukakis $C$ et al. Hyposmia and cognitive impairment in Gaucher disease patients and carriers. Mov Disord, 2012, 27(4):526-32.

10. Bultron G, Kacena K, Pearson D et al. The risk of Parkinson's disease in type 1 Gaucher disease. J Inherit Metab Dis, 2010. 33(2):167-73.

Revised: 02 January 2020, Accepted: 14 February 2020 